\title{
Dosimetric comparison of coplanar and non-coplanar volumetric-modulated arc therapy in head and neck cancer treated with radiotherapy
}

\author{
Sanjib Gayen, Sri Harsha Kombathula, Sumanta Manna, Sonal Varshney, Puneet Pareek \\ Department of Radiation Oncology, All India Institute of Medical Sciences, Jodhpur, India
}

Received: March 19, 2020

Revised: April 15, 2020

Accepted: April 22, 2020

Correspondence:

Sanjib Gayen,

Department of Radiation Oncology,

All India Institute of Medical

Sciences, Basni, Jodhpur, Rajasthan

342005, India.

Tel: +91-8369697171

E-mail: physanjib@gmail.com

ORCID:

https://orcid.org/0000-0003-3273-1134
Purpose: To evaluate the dosimetric variations in patients of head and neck cancer treated with definitive or adjuvant radiotherapy using optimized non-coplanar (ncVMAT) beams with coplanar (cVMAT) beams using volumetric arc therapy.

Materials and Methods: Twenty-two patients of head and neck cancer that had received radiotherapy using VMAT in our department were retrospectively analyzed. Each of the patients was planned using coplanar and non-coplanar orientations using an optimized couch angle and fluences. We analyzed the Conformity Index $\left(\mathrm{Cl}_{\text {RTOG }}\right)$, Dose Homogeneity Index (DHI), Heterogeneity Index $\left(\mathrm{HI}_{\mathrm{RTOG}}\right)$, low dose volume, target and organs-at-risk coverage in both the plans without changing planning optimization parameters.

Results: The prescription dose ranged from 60 Gy to $70 \mathrm{~Gy}$. Using ncVMAT, $\mathrm{Cl}_{\text {RTOG }}, \mathrm{DHI}$ and $\mathrm{HI}_{\text {RTOG }}$ and tumor coverage $\left(\mathrm{ID}_{95 \%}\right)$ had improved, low dose spillage volume in the body $\mathrm{V}_{5 \mathrm{~Gy}}$ was increased and $V_{10 G y}$ was reduced. Integral dose and intensity-modulated radiation therapy factor had increased in ncVMAT. In the case of non-coplanar beam arrangements, maximum dose $\left(D_{\max }\right)$ of right and left humeral head were reduced significantly whereas apex of the right and left lung mean dose were increased.

Conclusion: The use of ncVMAT produced better target coverage and sparing of the shoulder and soft tissue of the neck as well as the critical organ compared with the cVMAT in patients of head and neck malignancy.

Keywords: Head and neck neoplasm, Intensity-modulated radiotherapy

\section{Introduction}

Head and neck cancers form about 30\% of the total malignancies registered in a year in India. More than 400,000 new cases are diagnosed per annum [1,2]. Radiotherapy plays a major role in the management of this diverse spectrum of malignancies in the definitive, adjuvant and palliative setting. The major proportion of patients in India present in the advanced stages of the disease which further cements the position of radiotherapy in the plan of management. In the radical scheme of treatment of head and cancers, volumetric-modulated arc therapy (VMAT) was found to provide similar conformity and better homogeneity and organa-at-risk (OAR) sparing when compared to intensity-modulated radiation therapy (IMRT) or three-dimensional conformal radiation therapy [3-7]. Currently, considering the anatomical complexity, target coverage and OARs sparing, the use of non-coplanar beam arrangements with possible arc gantry geometry showed improvement over coplanar beam arrangements from various perspectives [8-10].

Owing to the normal anatomy of the human body, irradiation with coplanar VMAT (cVMAT) is different from non-coplanar VMAT 
(ncVMAT) due to longer photon path length of the coplanar beams passing through the shoulders and soft tissue of the neck to reach the target. The goal of radiotherapy is to maximize the dose to the primary site with adequate margins while minimizing dose to the nearby structures. Dosimetric comparisons between ncVMAT and cVMAT treatment plans showed clinically significant dosimetric improvements through an optimization process that could potentially allow substantial dose escalation, and improved local tumor control without exceeding critical OARs dose limits decided for the clinical plans for patients on conventional $\mathrm{C}$-arm linear accelerators [11-13].

With this background, we undertook a retrospective analysis of the patients of head and neck cancer treated in our center to study the dosimetric differences between the two commonly employed planning techniques, namely, coplanar and non-coplanar VMAT planning.

\section{Materials and Methods}

\section{Patients}

Plans of 22 patients of head and neck cancer that had received radiotherapy using VMAT in our department during 2018-2019 were retrospectively analyzed. All of them received definitive or adjuvant radiation therapy with curative intent up to a dose of 70 Gy delivered based on their clinical-stage, over 6-7 weeks ( 5 fractions per week) by a clinical linear accelerator (LINAC) (Elekta Versa HD; Elekta, Crawley, UK), following the International Commission on Radiation Units and Measurements (ICRU) recommendations, either alone or in combination with concomitant chemotherapy (chemoradiation).

\section{Simulation and contouring}

Each patient was positioned supine on a 16-slice computed tomography (CT) simulator (Optima 580; GE Healthcare, Waukesha, WI, USA) using a whole-body board (MacroMedics, Waddinxveen, The Netherlands) with a thermoplastic mask covering the head and shoulders and helical scans of $2.5 \mathrm{~mm}$ slice thickness were acquired from vertex to carina while the patient was breathing freely. The contours were done in the Monaco SIM (V5.11.02; Elekta CMS, Sunnyvale, CA, USA) contouring workstation.

Following the ICRU guidelines, the planning target volumes (PTV) of each of the patients included the primary with the lower limit of PTV including level IVA. The target volume delineation was done according to standard institutional protocol. The gross tumor volume to clinical target volume (CTV) was expanded by $1 \mathrm{~cm}$ in the low risk (CTV-low) along with the areas that are at low risk for the subclinical spread and by $0.5 \mathrm{~cm}$ along with the areas at high risk for the subclinical spread in high risk (CTV-high). The CTV to PTV expansion was $0.3 \mathrm{~cm}$ in all the cases. The OARs analyzed were bilateral humeral heads, bilateral lung apices, bilateral brachial plexuses, bilateral parotid glands, brainstem, and spinal cord. All the organs were contoured according to the Radiation Therapy Oncology Group (RTOG) atlas for normal tissue contouring [14]. The lung apices were contoured to a level that is $4 \mathrm{~cm}$ beyond the lowest level of PTV for uniformity among patients.

\section{Dose prescription}

Eleven patients were treated using the standard simultaneous integrated boost (SIB) technique. In this population, patients were prescribed up to a total dose of 70 Gy to the PTV-high risk and 54 Gy to the PTV-low risk with a maximum of 35 fractions.

Another set of 11 patients were treated using sequential (Phase I and Phase II) technique, in which the patients were prescribed up to $70 \mathrm{~Gy}$ in 35 fractions at $2 \mathrm{~Gy}$ per fraction.

In the sequential technique, all the patients were planned with both coplanar and non-coplanar beam arrangements in Phase I with dose ranging from $46 \mathrm{~Gy}$ to $60 \mathrm{~Gy}$ and the remaining dose was delivered in the next phase. In Phase II, PTV was located most of the time above the shoulder and the application of non-coplanar beams was not taken as a consideration. For this reason, we had taken only the sequential based Phase I and the dosimetric comparison had been reported between cVMAT and ncVMAT plans. Patient characteristics and dose prescriptions are described in Table 1.

\section{Radiotherapy planning and dosimetry}

All patients were planned using volumetric arc therapy treatment plans designed on Monaco (V5.11.02, Elekta CMS) treatment planning system with $6 \mathrm{MV}$ flat photon beam which has maximum dose rate of $600 \mathrm{cGy} / \mathrm{min}$ at $D_{\max }$. Each of the patients was planned using coplanar and non-coplanar orientations using an optimized couch angle and fluences. Both the cVMAT and ncVMAT plans were qualitatively evaluated for each patient and the dosimetric data was taken from the dose-volume histogram (DVH) data which represents the whole dose-volume information in a two-dimensional single curve.

Each of the non-coplanar beam angles in our approach was associated with achievable gantry-couch-patient clearance. Non-coplanar plans were generated using two non-coplanar beam arrangements with double arc VMAT in which, the gantry angles were chosen from $0^{\circ}$ to $+180^{\circ}$ with $350^{\circ}$ couch angle for the first beam arrangement in the clockwise direction and $0^{\circ}$ to $-180^{\circ}$ with $10^{\circ}$ couch angle in the counterclockwise direction for the second beam arrangement with an increment of $20^{\circ}$. For ncVMAT, we had 
Table 1. Patients characteristics

\begin{tabular}{|c|c|c|c|c|c|c|c|c|c|c|}
\hline & \multirow{2}{*}{ Patient\# } & \multirow{2}{*}{ Age (yr) } & \multirow{2}{*}{ Sex } & \multirow{2}{*}{ Primary } & \multirow{2}{*}{ Stage } & \multirow{2}{*}{ Intent } & \multicolumn{4}{|c|}{ Dose fractionation } \\
\hline & & & & & & & PTV-high & PTV-low & Phase I & Phase II \\
\hline \multirow[t]{11}{*}{ SIB } & 1 & 50 & $\mathrm{M}$ & Tongue & IVB & Adjuvant & 70 Gy in $35 \mathrm{fx}$ & $54 \mathrm{~Gy}$ in $35 \mathrm{fx}$ & - & - \\
\hline & 2 & 43 & $\mathrm{M}$ & Hypo-pharynx & IVA & Definitive & 70 Gy in 35 fx & $54 \mathrm{~Gy}$ in $35 \mathrm{fx}$ & - & - \\
\hline & 3 & 57 & $M$ & Cheek & IVB & Adjuvant & 70 Gy in 35 fx & $54 \mathrm{~Gy}$ in $35 \mathrm{fx}$ & - & - \\
\hline & 4 & 51 & M & Base of tongue & ॥ & Definitive & 70 Gy in $33 \mathrm{fx}$ & $54 \mathrm{~Gy}$ in $33 \mathrm{fx}$ & - & - \\
\hline & 5 & 73 & M & Base of tongue & IVA & Definitive & $66 \mathrm{~Gy}$ in $33 \mathrm{fx}$ & $54 \mathrm{~Gy}$ in $33 \mathrm{fx}$ & - & - \\
\hline & 6 & 73 & $\mathrm{~F}$ & Base of tongue & IVA & Definitive & $66 \mathrm{~Gy}$ in $33 \mathrm{fx}$ & $54 \mathrm{~Gy}$ in $33 \mathrm{fx}$ & - & - \\
\hline & 7 & 51 & M & Base of tongue & IVA & Definitive & $66 \mathrm{~Gy}$ in $33 \mathrm{fx}$ & $54 \mathrm{~Gy}$ in $33 \mathrm{fx}$ & - & - \\
\hline & 8 & 61 & $\mathrm{~F}$ & Tongue & III & Adjuvant & $66 \mathrm{~Gy}$ in $30 \mathrm{fx}$ & $54 \mathrm{~Gy}$ in $30 \mathrm{fx}$ & - & - \\
\hline & 9 & 71 & $M$ & Hypo-pharynx & III & Definitive & $66 \mathrm{~Gy}$ in $30 \mathrm{fx}$ & $54 \mathrm{~Gy}$ in $30 \mathrm{fx}$ & - & - \\
\hline & 10 & 75 & $\mathrm{~F}$ & Base of tongue & III & Definitive & 66 Gy in $30 \mathrm{fx}$ & $54 \mathrm{~Gy}$ in $30 \mathrm{fx}$ & - & - \\
\hline & 11 & 63 & $\mathrm{M}$ & Tongue & IVA & Adjuvant & $60 \mathrm{~Gy}$ in $30 \mathrm{fx}$ & $54 \mathrm{~Gy}$ in $30 \mathrm{fx}$ & - & - \\
\hline \multirow[t]{11}{*}{ Sequential } & 12 & 55 & $\mathrm{M}$ & Soft palate & IVA & Adjuvant & - & - & $54 \mathrm{~Gy}$ in $27 \mathrm{fx}$ & $12 \mathrm{~Gy}$ in $6 \mathrm{fx}$ \\
\hline & 13 & 76 & $M$ & Tongue & IVA & Adjuvant & - & - & $54 \mathrm{~Gy}$ in $27 \mathrm{fx}$ & $12 \mathrm{~Gy}$ in $6 \mathrm{fx}$ \\
\hline & 14 & 59 & $M$ & Oropharynx & IVA & Adjuvant & - & - & $54 \mathrm{~Gy}$ in $27 \mathrm{fx}$ & $16 \mathrm{~Gy}$ in $8 \mathrm{fx}$ \\
\hline & 15 & 42 & $\mathrm{~F}$ & Base of tongue & III & Definitive & - & - & 50 Gy in $25 \mathrm{fx}$ & $16 \mathrm{~Gy}$ in $8 \mathrm{fx}$ \\
\hline & 16 & 31 & M & Tongue & IVA & Adjuvant & - & - & 46 Gy in $23 \mathrm{fx}$ & $14 \mathrm{~Gy}$ in $7 \mathrm{fx}$ \\
\hline & 17 & 43 & $\mathrm{~F}$ & Base of tongue & IVA & Definitive & - & - & 46 Gy in $23 \mathrm{fx}$ & $14 \mathrm{~Gy}$ in $7 \mathrm{fx}$ \\
\hline & 18 & 33 & M & Cheek & IVB & Adjuvant & - & - & $54 \mathrm{~Gy}$ in $27 \mathrm{fx}$ & $16 \mathrm{~Gy}$ in $8 \mathrm{fx}$ \\
\hline & 19 & 24 & $\mathrm{~F}$ & Nasopharynx & IVA & Definitive & - & - & $60 \mathrm{~Gy}$ in $30 \mathrm{fx}$ & $10 \mathrm{~Gy}$ in $5 \mathrm{fx}$ \\
\hline & 20 & 46 & M & Cheek & IVB & Adjuvant & - & - & $54 \mathrm{~Gy}$ in $27 \mathrm{fx}$ & $16 \mathrm{~Gy}$ in $8 \mathrm{fx}$ \\
\hline & 21 & 62 & $\mathrm{~F}$ & Upper alveolus & $\|$ & Adjuvant & - & - & $54 \mathrm{~Gy}$ in $27 \mathrm{fx}$ & 16 Gy in $8 \mathrm{fx}$ \\
\hline & 22 & 49 & $\mathrm{M}$ & Tonsil & ॥ & Definitive & - & - & $54 \mathrm{~Gy}$ in $27 \mathrm{fx}$ & $16 \mathrm{~Gy}$ in $8 \mathrm{fx}$ \\
\hline
\end{tabular}

PTV, planning target volume; SIB, simultaneous integrated boost.

used partial arcs with a value of $\pm 10^{\circ}$ couch angle to avoid the shoulder region. The maximum number of control points per arc was selected as 180 to allow sufficient modulation and acceptable duration of the optimization with a minimum segment width of 1 $\mathrm{cm}$. The collimator angle was selected typically according to beam alignment to avoid tongue-and-groove effects and to cover the entire PTV region.

Similarly, coplanar plans were generated using single beam arrangement with double arc VMAT in which the gantry was chosen from $+180^{\circ}$ to $-180^{\circ}$ with $0^{\circ}$ couch angle and $0^{\circ}$ collimator angle in a counterclockwise direction or clockwise direction with a same increment angle $20^{\circ}$. All the other planning parameters were as same as ncVMAT plans. The typical beam arrangements for an example case is illustrated in Fig. 1.

The optimization technique used in VMAT is segment shape optimization (SSO) where the target dose rate will be auto-selected by the system itself. Similar to the sliding window technique, VMAT follows sweep sequencer and create segments and optimizes to achieve the desired dose distribution to target and OARs. The PTVs were reduced to $1 \mathrm{~mm}$ below the skin surface. Treatment plans were optimized with the same dose constraints for OAR. For PTV coverage, described by the ICRU Report 83, 95\% of PTV volume should receive $95 \%$ of the prescribed dose.

All the VMAT plans were created and calculated using the Monte Carlo (v1.6) dose calculation algorithm based on cost functions (achieving a dose distribution to the given value) with a grid size of $0.3 \mathrm{~cm}$ and $2 \%$ calculation uncertainty based on the beam data of our LINAC equipped with 160 leaves Agility MLC of $5 \mathrm{~mm}$ leaf thickness.

The Conformity Index $\left(\mathrm{Cl}_{\text {RTOG }}\right)$ [15-17], isodose line covering 95\% of the volume within PTV (ID $\left.{ }_{95 \%}\right)$, Dose Homogeneity Index (DHI) [15-17], Homogeneity Index ( $\left.\mathrm{HI}_{\mathrm{RTOG}}\right)$ [15-17], conformity number (CN) [15-18], low dose volume and OAR coverage in both the plans were analyzed. Total monitor units (MU) per fraction, IMRT ratio, i.e., total MU per cGy prescription dose [19-23], and the integral dose to patients $[24,25]$ were noted and compared. The dose covering a percentage of the structure's volume $\left(D_{\%}\right)$ and the volume of the structure receiving a certain dose $\left(\mathrm{V}_{\%}\right.$ or $\left.\mathrm{V}_{G y}\right)$ were used for dosimetric evaluation and planning purposes. For low dose volume, $V_{5 G y^{\prime}} V_{10 G y^{\prime}} V_{20 G y^{\prime}}$ and $V_{30 G y}$ were analyzed and compared. The OAR specific variables assessed were $D_{\max }$ and $D_{15 \%}$ for the humeral heads, $D_{\max }$ and $D_{5 \%}$ for the brachial plexuses, $D_{\text {mean }}$ for the lung 

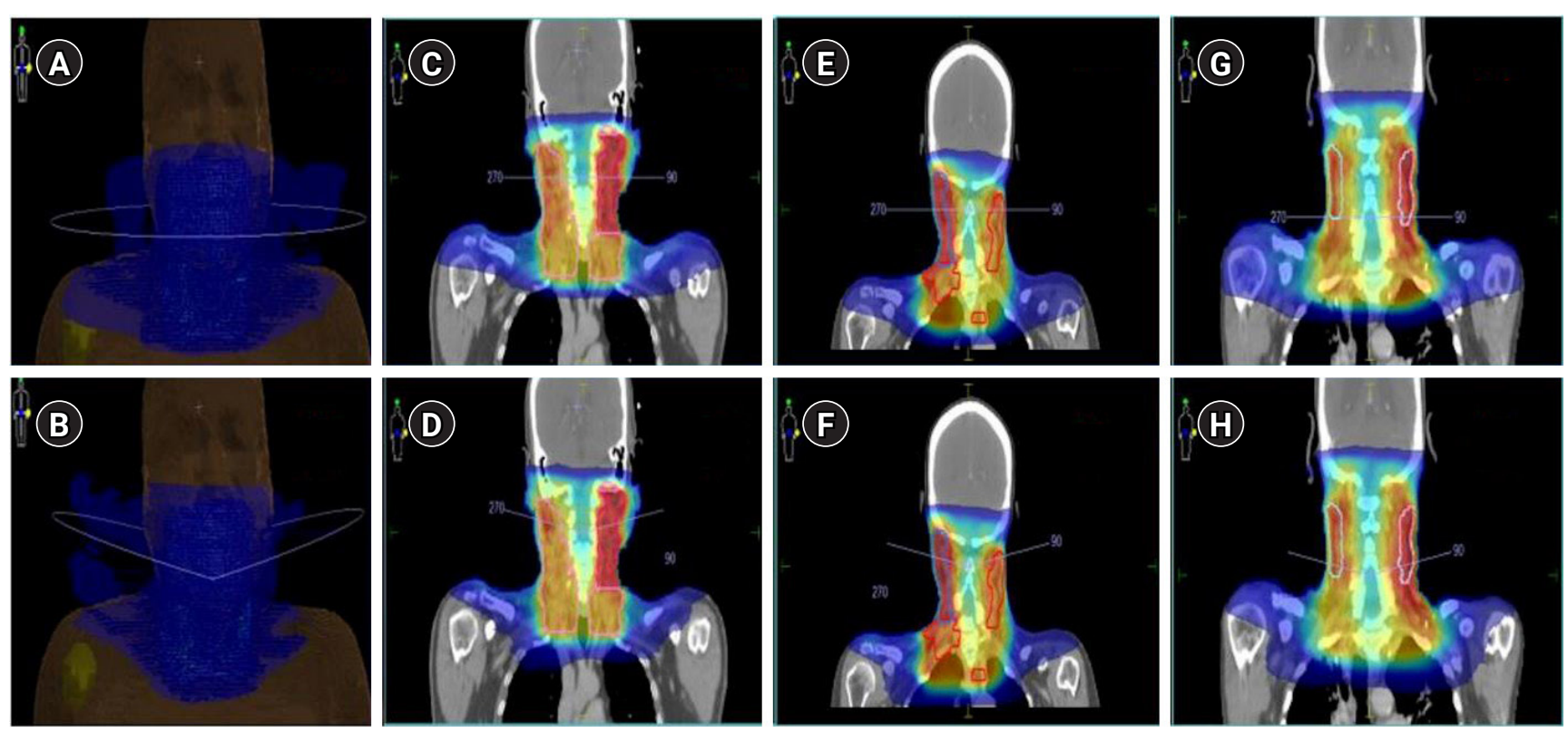

Fig. 1. The typical beam arrangements of coplanar VMAT plan (A) and non-coplanar VMAT plan (B) for an example case with a dose color wash. The typical dose distribution of both the beam arrangements in coronal planes for three example cases with a color-wash display ranging from $5 \mathrm{~Gy}$ to $70 \mathrm{~Gy}$ where $(\mathrm{C}),(\mathrm{E})$, and $(\mathrm{G})$ are the three coronal planes for coplanar beam arrangements and (D), (F), and (H) are the same three coronal planes for non-coplanar beam arrangements, respectively.

apices, $D_{\text {mean }}$ and $D_{50 \%}$ for the parotids and $D_{\max }$ for both the spinal cord and brainstem.

\section{Statistical analysis}

Statistical comparison of planning parameters, dose to the OARs and low dose volumes between cVMAT and ncVMAT plans were performed using SPSS, a data analysis software. As all the variables are quantitative, each of them is denoted by their respective means with standard deviation or with their ranges and analyzed statistically using the Wilcoxon matched paired signed-rank test. Evaluation of the level of significance of the observed difference between the dose-volume metrics had been performed and $p<0.05$ was considered as statistically significant.

\section{Results}

Of the 22 patients with a median age of 53 years analyzed, 11 patients were treated with adjuvant intent and 11 with definitive intent, details regarding the prescription dose and stage are tabulated in Table 1. In Tables 2 and 3, the DVH parameters for the PTV and OARs are mentioned with both the types of beam arrangements in VMAT plans for all the cases.

For the single PTV in phase-I planned with sequential technique, there was no significant difference in the values of $\mathrm{Cl}_{\mathrm{RTOG}}, \mathrm{CN}, \mathrm{ID}_{95 \%}$ $\mathrm{DHI}$, and $\mathrm{HI}_{\mathrm{RTOG}}$. On the contrary, significant differences were found in the SIB technique. In the PTV-high risk, the average values for the $\mathrm{Cl}_{\mathrm{RTOG}}, \mathrm{CN}, \mathrm{ID}_{95 \%}$, and $\mathrm{DHI}$ were reduced by $4.2 \%, 2.4 \%, 2.3 \%$, and $2.2 \%$, respectively, but $\mathrm{HI}_{\text {RTOG }}$ was increased by $1.7 \%$ in cVMAT. Similarly in the PTV-low risk, the average values for the $\mathrm{Cl}_{\text {RTOG }} \mathrm{ID}_{95 \%}$ and $\mathrm{DHI}$ were reduced by $2.1 \%, 2.4 \%$ and $2.4 \%$ and $\mathrm{HI}_{\mathrm{RTOG}}$ was increased by $2.2 \%$ in cVMAT. The monitor units utilized in the plans were significantly higher in the non-coplanar sequential plans but the same could not achieve statistical significance in the SIB technique. Treatment delivery time was significantly longer for non-coplanar plans in both sequential and SIB modalities. For all the cases, the average value of the IMRT ratio in the cVMAT plans was significantly reduced by $5.4 \%$.

Upon analysis of DVH parameters of the OARs achieved, it was noted that the $D_{\max }$ of the bilateral humeral head, $D_{\max }$ of right brachial plexus, and $D_{50 \%}$ of the left parotid were significantly higher in the cVMAT plans. At the same time, $D_{\text {mean }}$ of the bilateral lung apices was significantly lower in the cVMAT plans. For the right humeral head, the average value of $D_{\max }$ was increased by $50.7 \%$ in the sequential plans and $48.3 \%$ in the SIB plan, respectively. Similarly, for the left humeral head, the average value of $D_{\max }$ was increased by $42.2 \%$ in the sequential plans and $21.1 \%$ in the SIB plan, respectively. In the right brachial plexus, the average value of $D_{\max }$ was increased by $1.1 \%$ in the sequential plans and $1.2 \%$ in the SIB plan, respectively. In the left parotid, the average value of $D_{50 \%}$ was increased by $2 \%$ in the sequential plans and $5.7 \%$ in the SIB 
Table 2. Summary of the quantitative analysis of DVH of the target volumes for the two techniques

\begin{tabular}{|c|c|c|c|c|c|c|c|c|c|}
\hline & \multirow{2}{*}{\multicolumn{3}{|c|}{$\begin{array}{l}\text { Sequential } \\
\text { TV in phase-I }\end{array}$}} & \multicolumn{6}{|c|}{ SIB } \\
\hline & & & & \multicolumn{3}{|c|}{ PTV-high } & \multicolumn{3}{|c|}{ PTV-low } \\
\hline & ncVMAT & cVMAT & $p$-value & ncVMAT & cVMAT & $p$-value & ncVMAT & cVMAT & $p$-value \\
\hline CIRTOG & $0.95 \pm 0.02$ & $0.93 \pm 0.02$ & 0.159 & $0.96 \pm 0.02$ & $0.92 \pm 0.05$ & $0.007^{*}$ & $0.97 \pm 0.02$ & $0.95 \pm 0.02$ & $0.007^{*}$ \\
\hline $\mathrm{CN}$ & $0.75 \pm 0.03$ & $0.75 \pm 0.04$ & 0.435 & $0.83 \pm 0.06$ & $0.81 \pm 0.06$ & $0.031^{*}$ & $0.36 \pm 0.15$ & $0.36 \pm 0.15$ & 0.317 \\
\hline $\mathrm{ID}_{95 \%}$ & $94.2 \pm 0.99$ & $93.66 \pm 1.29$ & 0.125 & $95.40 \pm 1.12$ & $93.23 \pm 1.94$ & $0.006^{*}$ & $97.65 \pm 2.01$ & $95.30 \pm 1.91$ & $0.003^{*}$ \\
\hline $\mathrm{DHI}$ & $0.91 \pm 0.01$ & $0.90 \pm 0.01$ & $0.020^{*}$ & $0.92 \pm 0.01$ & $0.90 \pm 0.02$ & $0.005^{*}$ & $0.84 \pm 0.04$ & $0.82 \pm 0.04$ & $0.017^{*}$ \\
\hline $\mathrm{HI}_{\mathrm{RTOG}}$ & $1.18 \pm 0.02$ & $1.19 \pm 0.03$ & 0.11 & $1.16 \pm 0.02$ & $1.18 \pm 0.03$ & $0.028^{*}$ & $1.34 \pm 0.08$ & $1.37 \pm 0.08$ & $0.007^{*}$ \\
\hline MU & $900.02 \pm 94.31$ & $860.67 \pm 76.51$ & $0.003^{*}$ & - & - & - & $921.12 \pm 128.83$ & $860.55 \pm 86.71$ & 0.110 \\
\hline Delivery time (min) & $11.5 \pm 2.23$ & $10.69 \pm 2.33$ & $0.042^{*}$ & - & - & - & $12 \pm 2.12$ & $11.28 \pm 2.04$ & $0.043^{*}$ \\
\hline IMRT ratio (overall) & & & & $4.48 \pm 0.55$ & $4.24 \pm 0.43$ & 0.00 & & & \\
\hline
\end{tabular}

Values are presented as mean \pm standard deviation.

DVH, dose-volume histograms; PVT, planning target volume; ncVMAT, non-coplanar volumetric-modulated arc therapy; cVMAT, coplanar VMAT; Cl Conformity Index (according to RTOG); CN, conformity number; ID $_{95 \% \text {, }}$ isodose line covering $95 \%$ volume of the target; DHI, Dose Homogeneity Index; $\mathrm{HI}_{\mathrm{RTOG}}$, Homogeneity Index (according to RTOG); SIB, simultaneous integrated boost; MU, monitor unit; IMRT, intensity-modulated radiation therapy. ${ }^{*} \mathrm{p}<0.05$.

Table 3. Summary of the quantitative analysis of DVH of the organs-at-risk and low dose volumes in the patients for the two techniques

\begin{tabular}{|c|c|c|c|c|c|c|c|}
\hline \multirow{2}{*}{ Parameter } & & \multicolumn{3}{|c|}{ Sequential } & \multicolumn{3}{|c|}{ SIB } \\
\hline & & ncVMAT & cVMAT & $p$-value & ncVMAT & cVMAT & $p$-value \\
\hline \multirow[t]{2}{*}{ Rt. Humeral head } & $D_{15 \%}(G y)$ & $2.91 \pm 0.88$ & $3.62 \pm 2.12$ & 0.374 & $5.96 \pm 3.77$ & $7.79 \pm 4.59$ & 0.182 \\
\hline & $D_{\max }(G y)$ & $4.34 \pm 1.57$ & $6.54 \pm 3.03$ & $0.008^{*}$ & $9.07 \pm 5.74$ & $13.45 \pm 5.58$ & $0.007^{*}$ \\
\hline \multirow[t]{2}{*}{ Lt. Humeral head } & $\mathrm{D}_{15 \%}$ (Gy) & $3.97 \pm 1.18$ & $4.30 \pm 1.50$ & 0.534 & $5.71 \pm 2.32$ & $6.57 \pm 4.14$ & 0.374 \\
\hline & $\mathrm{D}_{\max }(\mathrm{Gy})$ & $5.74 \pm 1.53$ & $8.16 \pm 2.66$ & $0.050^{*}$ & $8.99 \pm 4.70$ & $10.89 \pm 5.24$ & $0.050^{*}$ \\
\hline \multirow[t]{2}{*}{ Lt. Brachial plexus } & $\mathrm{D}_{\max }(\mathrm{Gy})$ & $56.15 \pm 4.40$ & $56.42 \pm 4.09$ & 0.374 & $64.22 \pm 4.95$ & $64.74 \pm 4.81$ & 0.328 \\
\hline & $\mathrm{D}_{5 \%}(\mathrm{~Gy})$ & $53.75 \pm 4.27$ & $53.85 \pm 4.24$ & 0.45 & $59.95 \pm 4.31$ & $60.31 \pm 4.37$ & 0.328 \\
\hline \multirow[t]{2}{*}{ Rt. Brachial plexus } & $\mathrm{D}_{\max }(\mathrm{Gy})$ & $55.76 \pm 4.05$ & $56.37 \pm 4.25$ & $0.045^{*}$ & $63.15 \pm 4.45$ & $63.89 \pm 4.11$ & $0.041^{*}$ \\
\hline & $\mathrm{D}_{5 \%}(\mathrm{~Gy})$ & $53.56 \pm 4.08$ & $53.99 \pm 4.36$ & 0.059 & $59.72 \pm 4.23$ & $59.94 \pm 4.06$ & 0.594 \\
\hline Lt. Lung apex & $D_{\text {mean }}(G y)$ & $17.04 \pm 3.69$ & $15.13 \pm 4.14$ & $0.003^{*}$ & $18.24 \pm 3.99$ & $16.88 \pm 3.56$ & $0.040^{*}$ \\
\hline Rt. Lung apex & $\mathrm{D}_{\text {mean }}(\mathrm{Gy})$ & $16.14 \pm 3.16$ & $15.14 \pm 3.67$ & $0.004^{*}$ & $18.68 \pm 3.90$ & $17.02 \pm 3.70$ & $0.005^{*}$ \\
\hline Spinal cord & $\mathrm{D}_{\max }(\mathrm{Gy})$ & $35.03 \pm 2.58$ & $35.05 \pm 2.92$ & 0.374 & $40.53 \pm 1.78$ & $40.45 \pm 1.54$ & 0.657 \\
\hline Brainstem & $\mathrm{D}_{\max }(\mathrm{Gy})$ & $34.06 \pm 5.69$ & $33.94 \pm 4.13$ & 0.534 & $40.43 \pm 5.72$ & $39.29 \pm 6.42$ & 0.445 \\
\hline \multirow[t]{2}{*}{ Lt. Parotid } & $D_{\text {mean }}(G y)$ & $21.59 \pm 3.64$ & $21.85 \pm 3.50$ & 0.092 & $27.98 \pm 7.37$ & $28.39 \pm 8.02$ & 0.534 \\
\hline & $\mathrm{D}_{50 \%}(\mathrm{~Gy})$ & $15.80 \pm 5.77$ & $16.11 \pm 5.40$ & $0.002^{*}$ & $22.45 \pm 10.74$ & $23.74 \pm 11.59$ & $0.004^{*}$ \\
\hline \multirow[t]{2}{*}{ Rt. Parotid } & $\mathrm{D}_{\text {mean }}(\mathrm{Gy})$ & $21.40 \pm 2.39$ & $21.76 \pm 2.67$ & 0.241 & $31.71 \pm 9.01$ & $31.52 \pm 9.01$ & 0.929 \\
\hline & $D_{50 \%}(G y)$ & $14.12 \pm 2.09$ & $14.68 \pm 2.92$ & 0.203 & $28.83 \pm 16.16$ & $28.96 \pm 15.59$ & 1.000 \\
\hline Integral dose (Gy.L) & & $113.51 \pm 15.78$ & $110.77 \pm 14.52$ & $0.004^{*}$ & $129.13 \pm 21.02$ & $126.35 \pm 21.20$ & $0.004^{*}$ \\
\hline \multirow[t]{4}{*}{ Low dose volume } & $\mathrm{V}_{5 \mathrm{~Gy}}(\mathrm{~mL})$ & $3,751.52 \pm 359.40$ & $3,647.82 \pm 375.82$ & $0.033^{*}$ & $4,104.14 \pm 692.25$ & $3,963.03 \pm 732.43$ & $0.010^{*}$ \\
\hline & $\mathrm{V}_{10 \mathrm{~Gy}}(\mathrm{~mL})$ & $2,909.34 \pm 297.48$ & $2,943.97 \pm 288.88$ & $0.043^{*}$ & $3,133.32 \pm 568.04$ & $3,217.23 \pm 595.92$ & $0.040^{*}$ \\
\hline & $\mathrm{V}_{20 \mathrm{~Gy}}(\mathrm{~mL})$ & $2,181.05 \pm 300.21$ & $2,176.84 \pm 288.75$ & 0.424 & $2,304.10 \pm 417.52$ & $2,298.86 \pm 404.26$ & 0.929 \\
\hline & $V_{30 G y}(m L)$ & $1,584.82 \pm 250.35$ & $1,578.64 \pm 250.32$ & 1.000 & $1,704.40 \pm 327.61$ & $1,717.65 \pm 326.45$ & 0.286 \\
\hline
\end{tabular}

Values are presented as mean \pm standard deviation.

DVH, dose-volume histograms; SIB, simultaneous integrated boost; ncVMAT, non-coplanar volumetric-modulated arc therapy; cVMAT, coplanar VMAT; $D_{0 / 0}$ the dose covering a percentage of the structure's volume; $D_{\max }$ and $D_{\text {mean }}$ the maximum point dose and mean dose for a volume; $V_{G y}$ the volume of the structure receiving a certain dose.

${ }^{*} \mathrm{p}<0.05$. 

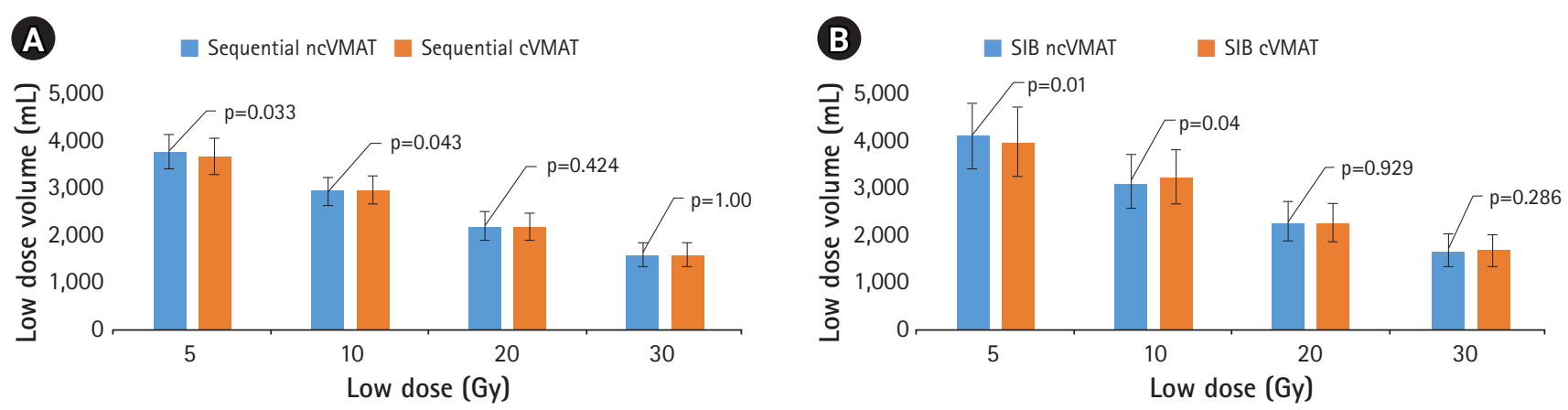

Fig. 2. Summary of the average values of volume encompassed by the low doses in the healthy body tissues for all the cases: (A) non-coplanar VMAT (ncVMAT) and coplanar VMAT (cVMAT) treatment plans for sequential based Phase I and (B) ncVMAT and cVMAT treatment plans for SIB technique. VMAT, volumetric-modulated arc therapy; SIB, simultaneous integrated boost.

plan, respectively. On the contrary, for left lung apex, the average value of $D_{\text {mean }}$ was simultaneously reduced by $11.2 \%$ in the sequential plans and $7.5 \%$ in the SIB plan, respectively. Similarly, for the right lung apex, the average value of $D_{\text {mean }}$ was simultaneously reduced by $6.2 \%$ in the sequential plans and $8.9 \%$ in the SIB plan, respectively. The rest of the dose parameters of the OARs assessed failed to show a statistically significant difference between the two types of plans. Fig. 1 depicts the typical dose distribution of both the beam arrangements in coronal planes for three example cases with a color-wash display ranging from 5 Gy to $70 \mathrm{~Gy}$. The analysis of the volume of the healthy tissue of body receiving low doses of the tune of $5 \mathrm{~Gy}, 10 \mathrm{~Gy}, 20 \mathrm{~Gy}$, and $30 \mathrm{~Gy}$ revealed that there was a statistically significant increase in $\mathrm{V}_{56 \mathrm{y}}$ by $2.8 \%$ in sequential and $3.4 \%$ in SIB techniques when planned on ncVMAT. Conversely, it showed a significant decrease in $\mathrm{V}_{10 \mathrm{~Gy}}$ by $1.2 \%$ in sequential and $2.7 \%$ in SIB techniques when planned on ncVMAT. The difference was not significant for higher doses like $\mathrm{V}_{20 \mathrm{~Gy}}$ and $\mathrm{V}_{30 \mathrm{~Gy}}$. In Fig. 2, the summary of the average values of volume encompassed by the low doses in the healthy body tissues for all the cases was depicted clearly. From the DVH metrics of all the cases, the average value of integral doses significantly reduced by $2.4 \%$ in sequential and $2.2 \%$ in SIB techniques when planned by cVMAT.

From the quantitative analysis of Fig. 3, it was noted the dose fall-off beyond the target region was similar for all the datasets. In our study, the decrease in the average value of total volume $(V)$ encompassed was following a logarithmic pattern for the increase in dose (D) value. For evaluation of the rate of dose fall-off beyond PTV, we have taken $\ln (1 / N)$ vs. In(D). From Fig. 4, it is represented that $\ln (1 / N)$ vs. $\ln (D)$ was following a straight line for all the different datasets. The gradient or slope was calculated from the graphs and the value of the gradient were 0.4647 and 0.4540 for ncVMAT and cVMAT (reduced by 2.3\%) in the sequential techniques and 0.4778 and 0.4606 for ncVMAT and cVMAT (reduced by $3.6 \%$ ) in

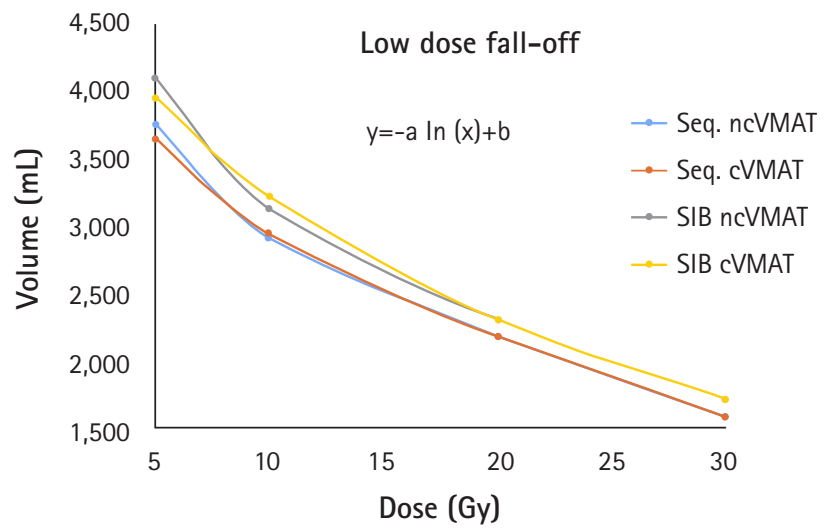

Fig. 3. The dose fall-off beyond the target region for all the cases planned with both the sequential and SIB technique for coplanar (cVMAT) and non-coplanar (ncVMAT) plans. Here, the y-axis represents the volume encompassed in cc and the $x$-axis represents the dose in Gy. For all the cases, in our study, the pattern of dose fall-off is best fitted by logarithmic curves with the equation written below $y=-a \ln (x)+b$, where $a$ and $b$ are constant values, respectively. VMAT, volumetric-modulated arc therapy; SIB, simultaneous integrated boost.

SIB techniques, respectively.

\section{Discussion and Conclusion}

Current treatment modalities introduced new radiotherapy techniques which improved the quality of treatment. IMRT is a widely used technique for head and neck cancers delivering a non-uniform dose from multiple angles to create a very conformal dose to targets with minimal complication to surrounding OARs $[26,27]$. In arc-based IMRT (VMAT), the intensity is modulated generating dose fluences throughout the gantry rotation with the aid of variable speed of gantry, MLC movements and dose rate with shorter delivery time than IMRT. 

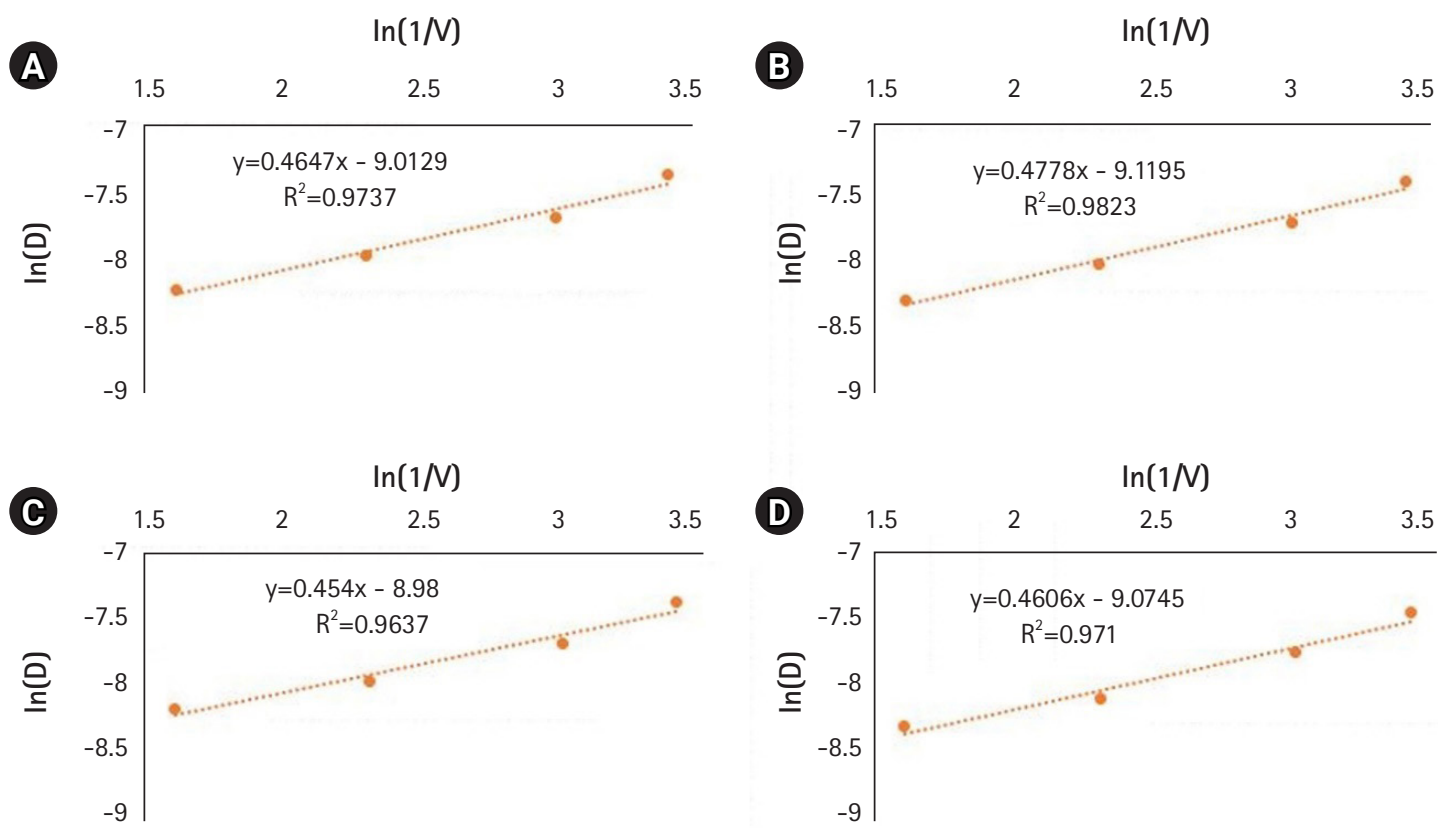

Fig. 4. The rate of dose fall-off beyond the target region by plotting $\ln (1 / N)$ vs. $\ln (D)$ in the $x$ and y axes, respectively, for all the cases planned with sequential ncVMAT (A), SIB ncVMAT (B), sequential cVMAT (C), and SIB cVMAT (D). cVMAT, coplanar volumetric-modulated arc therapy; ncVMAT, non-coplanar VMAT; SIB, simultaneous integrated boost.

In our study, the dosimetric advantage in the treatment of head and neck cancer patients was investigated and the robustness in the planning techniques was assessed by comparing ncVMAT and cVMAT in photon therapy. We found that the ncVMAT plan is more robust than the cVMAT plan for both target and normal tissues with increasing target coverage and conformity. Some studies indicate that coplanar beam arrangements in VMAT generate sub-optimal plans $[28,29]$. Non-coplanar arrangements of the beam with acceptable degrees of freedom generate better plans especially with regards to the OARs compared to coplanar beam arrangements. The improvement in the OAR dose parameters was observed with ncVMAT compared to cVMAT with other studies [30,31].

The delivery efficiency was assessed by choosing dose constraints to OARs and optimization parameters for both types of treatment plans [32]. In the daily treatment scenario for 6-7 weeks, the treatment delivery time is longer for ncVMAT. The benefit in coplanar beam arrangements is that gantry rotates in a smooth trajectory reducing overall treatment time and collision-free degrees of freedom. Furthermore, the range of MUs was larger and so was the IMRT ratio in the ncVMAT than cVMAT because of the complexity of beam optimization creating multiple apertures or segments of small field throughout different couch, collimator and gantry angles [33].

In our study, the same optimization parameter, i.e., number of arcs, gantry start and end angles, couch and collimator angles, number of control points per arc and minimum segment width were used in both of the sequential and SIB techniques for both types of the treatment plan. Therefore in the PTV for phase-I sequential technique, there was a non-significant change in the values of $\mathrm{Cl}_{\mathrm{RTOG}}, \mathrm{CN}, \mathrm{ID}_{95 \%}, \mathrm{DHI}$, and $\mathrm{HI}_{\mathrm{RTOG}}$ because of extra degrees of freedom which gives the optimizer additional space to reduce the dose to the OAR without reducing the dose to the target region for a comparably less prescription dose than SIB technique. On the other hand, in the SIB technique, all the dosimetric parameters for evaluation of target except CN in PTV-low showed significantly better results for ncVMAT.

Concerning OARs, for parotid glands, significant sparing was observed on $D_{50 \%}$ of the left parotid. The achievable dose in the parotid glands depends on the involvement in the PTV. The achieved $D_{\text {mean }}$ of the parotid glands was $>26$ Gy in our study as in certain other studies for the SIB plans $[34,35]$. For the spinal cord and brainstem, the achieved dose for $D_{\max }$ was $<45 \mathrm{~Gy}$ and $<54 \mathrm{~Gy}$ for all the planning techniques and a non-significant dose reduction was observed in the ncVMAT comparably from cVMAT. In brachial plexus, $D_{\max }$ should be < 66 Gy for head and neck cases in terms of plexopathy. In our study, dose constraints for bilateral brachial plexuses were achieved. A significant dose reduction was observed on $D_{\max }$ for the right brachial plexus [36-38].

A significant dose reduction was observed for bilateral lung apices in the cVMAT plans. The reason behind the increase in dose in 
bilateral lung apices is the exiting path which was directly through the lung apex regions. $D_{\text {mean }}$ for bilateral lung apices was reduced by nearly 2 Gy based on the overall prescription dose in the coplanar beam arrangements.

Irradiation of tumors in the head and neck cancer patients is technically challenging especially in the patients with a short neck or high shoulders. Considering patient comfort, we had fixed the patient position by applying a mask. Higher stage head neck cases required comprehensive irradiation of the neck region extending inferiorly to the level of the lung apices. Owing to the body structure, if we planned with coplanar beam arrangements, dose to the healthy tissue of the shoulder region would be higher than non-coplanar beam arrangements because of the photon path length to the PTV, i.e., photon beams had to pass through the shoulders and soft tissue of neck region [39]. For this reason, we evaluated the dose for bilateral humeral heads. A significant dose reduction was noted for $D_{\max }$ of bilateral humeral heads in non-coplanar beam arrangements.

The use of non-coplanar beam arrangements resulted in a broader dose bath because of quantitatively more irradiated volume in the healthy tissue. For that reason, the clinical relevance of the dose bath has to be considered carefully. A significant increase in integral dose in the patient body was observed although the difference was very less. When evaluating the lower dose volume in the healthy body tissues we observed that $\mathrm{V}_{5 \mathrm{~Gy}}$ was increased and conversely $\mathrm{V}_{10 \mathrm{~Gy}}$ was reduced in ncVMAT.

Due to the contributions of patient and collimator scatter, the energy spectrum is softer outside the treatment field than within the target volume $[40,41]$. The amount of low dose volume outside the treatment field is high in VMAT plans due to the distance from the field edge to the beam entry point through the body surface. The dose outside the treatment field also depends on the size of the target, increasing with increasing target volume because larger irradiated volumes produce more patient scatter in the body [42]. Gradually decrease in the average volume $(V)$ encompassed by a certain dose (D) was following a logarithmic pattern for an increase in the dose [43-46]. As depicted in Fig. 4, the rate of dose fall-off was higher in the non-coplanar beam arrangement than coplanar because of the high number of degrees of freedom available in the optimization process restricting the dose to the nearby OARs and healthy soft tissues of the patient body. The amount of intensity modulation was higher in the non-coplanar beam arrangements because the optimization was done with different beam angles according to the couch position with gantry-collimator adjustment [47].

In conclusion, VMAT plans with non-coplanar beam arrangements showed significant dosimetric advantages both on target coverage and OAR sparing compared with coplanar beam arrange- ments in the treatment of head and neck malignancy. However, there was a significant increase in MU, total delivery time, uncertainty in proper patient positioning, integral dose to the patient body, and dose to the bilateral lung apices in the ncVMAT plans. Conversely, there was a significant improvement in plan robustness, target conformity, low dose to the healthy tissues of shoulders and neck region, and both bilateral brachial plexus and humeral heads. One of the late toxicity in head and neck patients with contribution from both, surgery of neck as well as intermediate to low dose deposition by radiation therapy in neck and shoulders, results in fibrosis and reduced range of motion of shoulder joint and neck. The dose reduction to the shoulder joint and lower neck region by ncVMAT may translate in lesser late fibrosis of the same and maybe further studied clinically. The minimally increased dose deposition in ncVMAT to lung apices may not contribute to any increased pulmonary toxicity and may also be clinically confirmed at the same time.

\section{Conflict of Interest}

No potential conflict of interest relevant to this article was reported.

\section{References}

1. Tuljapurkar V, Dhar H, Mishra A, Chakraborti S, Chaturvedi P, Pai PS. The Indian scenario of head and neck oncology: challenging the dogmas. South Asian J Cancer 2016;5:105-10.

2. Mehrotra $R$, Singh $M$, Gupta RK, Singh $M$, Kapoor AK. Trends of prevalence and pathological spectrum of head and neck cancers in North India. Indian J Cancer 2005;42:89-93.

3. Vanetti $E_{1}$ Clivio A, Nicolini G, et al. Volumetric modulated arc radiotherapy for carcinomas of the oro-pharynx, hypo-pharynx and larynx: a treatment planning comparison with fixed field IMRT. Radiother Oncol 2009;92:111-7.

4. Verbakel WF, Cuijpers JP, Hoffmans D, Bieker M, Slotman BJ, Senan S. Volumetric intensity-modulated arc therapy vs. conventional IMRT in head-and-neck cancer: a comparative planning and dosimetric study. Int J Radiat Oncol Biol Phys 2009;74:2529.

5. Scorsetti M, Fogliata A, Castiglioni S, et al. Early clinical experience with volumetric modulated arc therapy in head and neck cancer patients. Radiat Oncol 2010;5:93.

6. Smet S, Lambrecht M, Vanstraelen B, Nuyts S. Clinical and dosimetric evaluation of RapidArc versus standard sliding window IMRT in the treatment of head and neck cancer. Strahlenther Onkol 2015;191:43-50. 
7. Fung-Kee-Fung SD, Hackett $R$, Hales L, Warren G, Singh AK. A prospective trial of volumetric intensity-modulated arc therapy vs conventional intensity modulated radiation therapy in advanced head and neck cancer. World J Clin Oncol 2012;3:57-62.

8. Wild E, Bangert M, Nill S, Oelfke U. Noncoplanar VMAT for nasopharyngeal tumors: plan quality versus treatment time. Med Phys 2015;42:2157-68.

9. Yu W, Tran A, Nguyen D, et al. The development and verification of a highly accurate collision prediction model for automated noncoplanar plan delivery. Med Phys 2015;42:6457-67.

10. Bangert $M$, Oelfke U. Spherical cluster analysis for beam angle optimization in intensity-modulated radiation therapy treatment planning. Phys Med Biol 2010;55:6023-37.

11. Yang $Y$, Zhang $P$, Happersett $L$, et al. Choreographing couch and collimator in volumetric modulated arc therapy. Int J Radiat Oncol Biol Phys 2011;80:1238-47.

12. Krayenbuehl J, Davis JB, Ciernik IF. Dynamic intensity-modulated non-coplanar arc radiotherapy (INCA) for head and neck cancer. Radiother Oncol 2006;81:151-7.

13. Voet PW, Breedveld S, Dirkx ML, Levendag PC, Heijmen BJ. Integrated multicriterial optimization of beam angles and intensity profiles for coplanar and noncoplanar head and neck IMRT and implications for VMAT. Med Phys 2012;39:4858-65.

14. Brouwer CL, Steenbakkers RJ, Bourhis J, et al. CT-based delineation of organs at risk in the head and neck region: DAHANCA, EORTC, GORTEC, HKNPCSG, NCIC CTG, NCRI, NRG Oncology and TROG consensus guidelines. Radiother Oncol 2015;117:83-90.

15. Kataria T, Sharma K, Subramani V, Karrthick KP, Bisht SS. Homogeneity Index: an objective tool for assessment of conformal radiation treatments. J Med Phys 2012;37:207-13.

16. Petrova D, Smickovska S, Lazarevska E. Conformity Index and Homogeneity Index of the postoperative whole breast radiotherapy. Open Access Maced J Med Sci 2017;5:736-9.

17. Cao T, Dai Z, Ding Z, Li W, Quan H. Analysis of different evaluation indexes for prostate stereotactic body radiation therapy plans: conformity index, homogeneity index and gradient index. Precis Radiat Oncol 2019;3:72-9.

18. Paddick I, Lippitz B. A simple dose gradient measurement tool to complement the conformity index. J Neurosurg 2006;105 Suppl:194-201.

19. Webb S. Conformal intensity-modulated radiotherapy (IMRT) delivered by robotic linac: conformality versus efficiency of dose delivery. Phys Med Biol 2000;45:1715-30.

20. Hauri P, Schneider U. Whole-body dose equivalent including neutrons is similar for $6 \mathrm{MV}$ and $15 \mathrm{MV}$ IMRT, VMAT, and 3D conformal radiotherapy. J Appl Clin Med Phys 2019;20:56-70.

21. Chow JC, Wettlaufer $B$, Jiang R. Dosimetric effects on the pen- umbra region of irregular multi-leaf collimated fields. Phys Med Biol 2006:51:N31-8.

22. Patel I, Glendinning AG, Kirby MC. Dosimetric characteristics of the Elekta Beam Modulator. Phys Med Biol 2005;50:5479-92.

23. Taylor ML, Kron T. Consideration of the radiation dose delivered away from the treatment field to patients in radiotherapy. J Med Phys 2011;36:59-71.

24. Aoyama H, Westerly DC, Mackie TR, et al. Integral radiation dose to normal structures with conformal external beam radiation. Int J Radiat Oncol Biol Phys 2006;64:962-7.

25. Slosarek K, Osewski W, Grzadziel A, et al. Integral dose: comparison between four techniques for prostate radiotherapy. Rep Pract Oncol Radiother 2014;20:99-103.

26. Kathirvel M, Subramani V, Subramanian VS, Swamy ST, Arun G, Kala S. Dosimetric comparison of head and neck cancer patients planned with multivendor volumetric modulated arc therapy technology. J Cancer Res Ther 2017;13:122-30.

27. Whitton $A$, Warde $P$, Sharpe $M$, et al. Organisational standards for the delivery of intensity-modulated radiation therapy in Ontario. Clin Oncol (R Coll Radiol) 2009;21:192-203.

28. Sheng K, Shepard DM, Orton CG. Noncoplanar beams improve dosimetry quality for extracranial intensity modulated radiotherapy and should be used more extensively. Med Phys 2015;42: $531-3$

29. Dong $P$, Lee $P$, Ruan $D$, et al. $4 \pi$ noncoplanar stereotactic body radiation therapy for centrally located or larger lung tumors. Int J Radiat Oncol Biol Phys 2013;86:407-13.

30. Hirashima $H$, Nakamura M, Miyabe $Y$, et al. Quality assurance of non-coplanar, volumetric-modulated arc therapy employing a C-arm linear accelerator, featuring continuous patient couch rotation. Radiat Oncol 2019;14:62.

31. Serre A, Idri K, Fenoglietto $P$, et al. Dosimetric comparison between coplanar and non coplanar field radiotherapy for ethmoid sinus cancer. Radiat Oncol 2007;2:35.

32. Lee TF, Ting HM, Chao PJ, Fang FM. Dual arc volumetric-modulated arc radiotherapy (VMAT) of nasopharyngeal carcinomas: a simultaneous integrated boost treatment plan comparison with intensity-modulated radiotherapies and single arc VMAT. Clin Oncol (R Coll Radiol) 2012;24:196-207.

33. Xia P, Verhey $\amalg$. Multileaf collimator leaf sequencing algorithm for intensity modulated beams with multiple static segments. Med Phys 1998;25:1424-34.

34. Nithya L, Raj NA, Kumar A, Rathinamuthu S, Pandey MB. Comparative analysis of volumetric-modulated arc therapy and intensity-modulated radiotherapy for base of tongue cancer. J Med Phys 2014;39:121-6.

35. Zheng BM, Dong XX, Wu H, Duan YJ, Han SK, Sun Y. Dosimetry 
comparison between volumetric modulated arc therapy with RapidArc and fixed field dynamic IMRT for local-regionally advanced nasopharyngeal carcinoma. Chin J Cancer Res 2011; 23:259-64.

36. Emami B, Lyman J, Brown A, et al. Tolerance of normal tissue to therapeutic irradiation. Int J Radiat Oncol Biol Phys 1991; 21:109-22.

37. Cox JD, Stetz J, Pajak TF. Toxicity criteria of the Radiation Therapy Oncology Group (RTOG) and the European Organization for Research and Treatment of Cancer (EORTC). Int J Radiat Oncol Biol Phys 1995;31:1341-6.

38. Lee S, Cao YJ, Kim CY. Physical and radiobiological evaluation of radiotherapy treatment plan. In : Nenoi $M$, editor. Evolution of ionizing radiation research Rijeka, Croatia: InTech; 2015, p. 10950.

39. Newbold KL, Bhide S, Convery H, Harrington KJ, Nutting CM. Prospective intra-patient evaluation of a shoulder retraction device for radiotherapy in head and neck cancer. Med Dosim 2012; 37:293-5.

40. Scarboro SB, Followill DS, Howell RM, Kry SF. Variations in photon energy spectra of a 6 MV beam and their impact on TLD re- sponse. Med Phys 2011;38:2619-28.

41. Edwards CR, Mountford PJ. Near surface photon energy spectra outside a 6 MV field edge. Phys Med Biol 2004;49:N293-301.

42. Stovall M, Blackwell CR, Cundiff J, et al. Fetal dose from radiotherapy with photon beams: report of AAPM Radiation Therapy Committee Task Group No. 36. Med Phys 1995;22:63-82.

43. Blais AR, Lederer $E_{1}$ Oliver M, Leszczynski K. Static and rotational step-and-shoot IMRT treatment plans for the prostate: a risk comparison study. Med Phys 2012;39:1069-78.

44. Petti PL, Chuang CF, Smith V, Larson DA. Peripheral doses in CyberKnife radiosurgery. Med Phys 2006;33:1770-9.

45. Ramsey C, Seibert R, Mahan SL, Desai D, Chase D. Out-of-field dosimetry measurements for a helical tomotherapy system. J Appl Clin Med Phys 2006;7:1-11.

46. Kragl G, Baier F, Lutz S, et al. Flattening filter free beams in SBRT and IMRT: dosimetric assessment of peripheral doses. Z Med Phys 2011;21:91-101.

47. Fogliata A, Wang PM, Belosi F, et al. Assessment of a model based optimization engine for volumetric modulated arc therapy for patients with advanced hepatocellular cancer. Radiat Oncol 2014;9:236. 